\title{
Density and energy relaxation in an open one-dimensional system
}

\author{
Prasanth P. Jose ${ }^{\text {a) }}$ and Biman Bagchi ${ }^{\text {b) }}$ \\ Solid State and Structural Chemistry Unit, Indian Institute of Science, Bangalore-560012, India
}

\begin{abstract}
A new master equation to mimic the dynamics of a collection of interacting random walkers in an open system is proposed and solved numerically. In this model, the random walkers interact through excluded volume interaction (single-file system); and the total number of walkers in the lattice can fluctuate because of exchange with a bath. In addition, the movement of the random walkers is biased by an external perturbation. Two models for the latter are considered: (1) an inverse potential $(V \propto 1 / r)$, where $r$ is the distance between the center of the perturbation and the random walker and (2) an inverse of sixth power potential $\left(V \propto 1 / r^{6}\right)$. The calculated density of the walkers and the total energy show interesting dynamics. When the size of the system is comparable to the range of the perturbing field, the energy relaxation is found to be highly nonexponential. In this range, the system can show stretched exponential $\left(e^{-\left(t / \tau_{s}\right)^{\beta}}\right)$ and even logarithmic time dependence of energy relaxation over a limited range of time. Introduction of density exchange in the lattice markedly weakens this nonexponentiality of the relaxation function, irrespective of the nature of perturbation.
\end{abstract}

\section{INTRODUCTION}

Relaxation dynamics of interacting particles in a onedimensional system is often difficult to understand because the traditional coarse-grained descriptions (such as hydrodynamics or time dependent mean-field type approximations) fail in this case. This is because of the existence of longrange correlations mediated through the excluded volume interaction. In such cases, random walk models have often proved to be successful in describing the nonexponential relaxation commonly observed in one-dimensional systems.

Recently, several experimental studies have reported energy relaxation in one-dimensional or quasi-one-dimensional systems such as DNA. ${ }^{1,2}$ These experiments employed the time dependent fluorescence Stokes shift technique to gather a quantitative measure of the time scale involved. In one of these experiments Bruns et al. ${ }^{1}$ have calculated structural relaxation of DNA oligonucleotides. They found that the redshift of the fluorescent spectrum follows a logarithmic time dependence. The above-described experiment is an example where dimensionality plays an important role in the energy and density relaxation.

In this work, we propose a random walk model for the carriers in a one-dimensional channel to mimic the relaxation of energy and density found in the above-described experiments. There were several theoretical studies devoted to random walk model in one-dimensional systems. ${ }^{3-9}$ Many recent studies based on random walk model in onedimensional systems have explored transport and other dynamical properties such as de conductivity, frequency dependent conductivity, effect of bias on diffusion, space and time dependent probability distribution of random walkers,

\footnotetext{
${ }^{a)}$ Electronic mail: jose@sscu.iisc.ernet.in

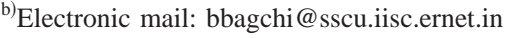

mean residence time, mean first passage time of random walkers, etc. ${ }^{9-16}$

The work presented here is based on a master equation for random walk in a single-file system (a one-dimensional system that does not allow particles to pass through each other) that allows exchange of the particles with the bath (see Fig. 1 for a schematic illustration). Here we employed two model potentials with different characteristics to study the effect of perturbation on the density and energy relaxation processes. The potentials used here are (1) inverse of distance potential (example is a Coulomb's field generated by a trapped charge in the lattice) and (2) inverse of sixth power of distance potential (this is a short range interaction compared to Coulomb interaction and is taken as attractive part of the Lennard-Jones interaction). Energy and density relaxation functions in a one-dimensional channel without number fluctuation is calculated and then compared with that of a channel, where particle number fluctuates due to exchange with the bath. It is observed that density fluctuation in the channel makes energy relaxation exponential.

The rest of this paper is organized as follows. Section II gives the description of the master equation used in the simulation. The details of the Monte Carlo simulations are given in Sec. III. Results of the simulations were analyzed in Sec. IV. Section V presents the conclusions from the Monte Carlo simulations.

\section{THE GENERALIZED MASTER EQUATION}

Let the total number of lattice sites in a system consisting of a linear lattice and a bath be $\mathcal{N}_{T}=\mathcal{N}_{L}+\mathcal{N}_{B}$, where $\mathcal{N}_{L}$ is the number of lattice sites of the lattice and $\mathcal{N}_{B}$ is the number of accessible bath sites. The time dependent probability $P_{i}^{L}(t)$ of finding a particle in the $i$ th site of the lattice at time $t$ is given by the following master equation: 


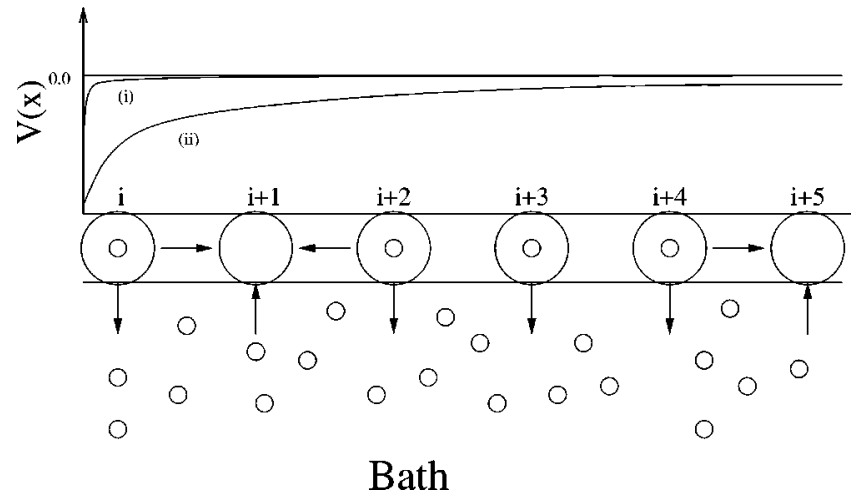

FIG. 1. The perturbation potential and the various transitions are shown schematically. The attractive part of the Lennard-Jones potential is shown by the line (i) and Coulomb potential is given by the line (ii).

$$
\begin{aligned}
\frac{d P_{i}^{L}(t)}{d t}= & \sum_{m=1}^{\mathcal{N}_{L}}{ }^{\prime} w_{m, i}(t) P_{m}^{L}(t)-w_{i, m}(t) P_{i}^{L}(t) \\
& -P_{i}^{L}(t) \sum_{j=1}^{\mathcal{N}_{B}} w_{i, j}(t)+\sum_{j=1}^{\mathcal{N}_{B}} w_{j, i}(t) P_{j}^{B}(t)
\end{aligned}
$$

(the prime on the summation signifies that the term $i=m$ is to be omitted from the sum), where $w_{m, i}(t)$ gives the transition probability of the particle from site $m$ to $i$ per unit time. The last two terms of the master equation introduce density fluctuation in the lattice by allowing exchange of particles with the bath. In the master equation, the index $j$ sums over the bath sites and $P_{j}^{B}$ is the probability of finding a particle in the $j$ th bath site. This master equation can be simplified by assuming that lattice sites in the bath are numerous $\left(\mathcal{N}_{B}\right.$ $\gg \mathcal{N}_{L}$ ) and the number of walkers in the bath is much larger than that in the lattice. These assumptions allow us to perform an averaging over the bath sites. Summation in the last two terms of Eq. (2) can be omitted by substituting the properties of the bath with that of an ideal bath defined in the following. The sum of the transition probability $w_{i, j}(t)$ to the bath sites can be replaced by the term $w_{\text {out }}(t)\left(\sum_{j=1}^{\mathcal{N}_{B}} w_{i, j}(t)\right.$ $\left.=w_{\text {out }}(t)\right)$, since $w_{i, j}(t)$ depends only on the temperature, size, and number of particles in the system (discussed in detail later in this section). This ideal bath has infinite capacity to absorb particles. Similarly, when a particle is absorbed by the lattice this bath site acts as a supplier of infinite number of particles. The transition probabilities from all lattice sites to the bath are equal and assumed to be independent of the instantaneous state of the bath. Therefore, $w_{j, i}$ can be replaced by $w_{\text {in }}$ and $\sum_{j=1}^{\mathcal{N}_{B}} P_{j}^{B}$ can be replaced by $P_{\text {bath }}$. The resultant master equation is simpler and is given by

$$
\begin{aligned}
\frac{d P_{i}^{L}(t)}{d t}= & \sum_{m}^{\mathcal{N}_{L}}{ }^{\prime} w_{m, i}(t) P_{m}^{L}(t)-w_{i, m}(t) P_{i}^{L}(t)-w_{\mathrm{out}} P_{i}^{L}(t) \\
& +w_{\text {in }}(t) P_{\mathrm{bath}} .
\end{aligned}
$$

If only nearest-neighbor exchanges are allowed then the master equation can be written as

$$
\begin{aligned}
\frac{d P_{i}^{L}(t)}{d t}= & w_{i+1, i}(t) P_{i+1}^{L}(t)+w_{i-1, i}(t) P_{i-1}^{L}(t) \\
& -w_{i, i+1}(t) P_{i}^{L}(t)-w_{i, i-1}(t) P_{i}^{L}(t) \\
& -w_{\text {out }}(t) P_{i}^{L}(t)+w_{\text {in }}(t) P_{\text {bath }} .
\end{aligned}
$$

At equilibrium this system represents a grand canonical ensemble. Hence for insertion and removal of the particles in the randomly selected sites we use grand-canonical Monte Carlo method, where probability $\left(\mathbf{P}_{\mu, L, T}\right)$ of system having number of particles $N$ is ${ }^{17}$

$$
\mathbf{P}_{\mu, L, T} \propto \frac{L^{N}}{\Lambda^{N} N !} e^{-\beta[E-\mu N]},
$$

where $\beta$ is the inverse of Boltzmann constant times absolute temperature $\left(1 / k_{B} T\right), \Lambda\left(=\sqrt{h^{2} / 2 \pi m k_{B} T}\right)$ is the thermal de Broglie wavelength, $L$ is the length of the linear lattice, $\mu$ is chemical potential, and $E$ is the potential energy of the linear lattice. Particles of this system obey Boltzmann distribution at equilibrium, hence the transition probability $w_{i, i+1}(t)$ can be calculated from the total energy cost for the move. The hopping probability is calculated for a particle from one site to another using Metropolis scheme. ${ }^{17}$ Hopping probability of a random walker from one site to the neighboring site of the lattice is given by

$$
w_{i, i+1}=\min \left[1, e^{-\beta \Delta E}\right] \text {. }
$$

The transition probabilities for absorption and desorption from the lattice can be obtained ${ }^{17}$ so as to satisfy the detailed balance at equilibrium as

$$
\begin{aligned}
& w_{\text {in }}=\min \left[1, \frac{Q}{(N+1)} e^{-\beta \Delta E}\right], \\
& w_{\text {out }}=\min \left[1, \frac{N}{Q} e^{\beta \Delta E}\right],
\end{aligned}
$$

where $Q$ is given by $(L / \Lambda) e^{\beta \mu}$. A nontrivial aspect of this master equation is that the transition probability $w_{\text {in }}(t)$ varies with time in response to the number fluctuations. This explicit time dependence of $w_{\text {in }}(t)$ poses formidable difficulty in obtaining an explicit analytic solution of the problem. In this system of interacting particles, the transition probability to neighboring sites depends on the instantaneous probability of that site being occupied. In a one-dimensional channel with hard rod interactions between the carriers, mobility of each carrier is restricted to a portion of the lattice. The particles move in the linear lattice under the influence of a chosen potential, which is at a fixed position of this lattice. Hence the energy cost for hopping in the lattice is

$$
\Delta E= \begin{cases}E_{1} & (\text { transition to vacant site }) \\ \infty & (\text { transition to occupied site })\end{cases}
$$

where

$$
E_{1}=K_{1}\left(\frac{1}{x_{i}}-\frac{1}{x_{i+1}}\right)
$$

for potential 1 and 


$$
E_{1}=K_{2}\left(\frac{1}{x_{i}^{6}}-\frac{1}{x_{i+1}^{6}}\right)
$$

for potential 2. For absorption of particles to the lattice at $i$ th site

$$
\Delta E= \begin{cases}E_{2} & (\text { creation of vacant site }) \\ \infty & (\text { creation of occupied site }),\end{cases}
$$

where $E_{2}=K_{1}\left(1 / x_{i}\right)$ for potential 1 and $E_{2}=K_{2}\left(1 / x_{i}^{6}\right)$ for potential 2. For the desorption of particles from the $i$ th site

$$
\Delta E=E_{2} .
$$

$Q$ in this system is constant and equal to the initial number of the particles in the lattice. This assumption specifies the value of the chemical potential of this linear lattice.

\section{THE DETAILS OF MONTE CARLO SIMULATION}

The Monte Carlo simulations are carried out on a linear lattice, with $50 \%$ (on average) of the lattice sites occupied by the random walkers. Closed boundary condition is used to explore the size effect on the relaxation. In this onedimensional lattice, the adjacent sites are equally spaced and all the sites are identical in energy in the absence of the perturbation. Here inhomogeneity in the lattice is generated by the perturbation of potential introduced in the lattice. In addition, there is a dynamic disorder in the lattice that originates from the instantaneous rearrangement of the interacting random walkers. The initial configuration of the random walkers in an unperturbed lattice is chosen from a random distribution, such that no two particles occupy the same site. The inverse potential arise from Coulomb interaction, hence the magnitude of the biasing potential is calculated from the interaction between the charge of the carriers $\left(q_{1}\right)$ and the charge at the center of bias $\left(q_{2}\right)$ in a medium of dielectric constant $\epsilon$. The constant $K_{1}$ for potential energy can be calculated as $K_{1}=q_{1} q_{2} / \epsilon$. Here both the perturbing charge and the charge of the carrier have magnitude of one electron and their signs are opposite. Assuming high screening effect, the value of $\epsilon$ is taken as equal to that of water, that is 80 . The distance between the adjacent sites in the linear lattice is $4 \AA$ (approximately the vertical distance between two DNA base pairs in the double helix). All simulations were conducted near room temperature $(300 \mathrm{~K})$. The value of $K_{1}$ used in the simulation is $-1.7 k_{B} T$. Compared to Coulomb's interaction, the Lennard-Jones interaction is short ranged, hence the constant $K_{2}$ is kept high to extend the range of the potential, value of $K_{2}$ used is $-10 k_{B} T$.

The nonequilibrium Monte Carlo simulation starts from a randomly chosen initial configuration. Then a perturbing potential is introduced in the lattice at at time $t=0$. Subsequent relaxation of energy is monitored. The simulation is repeated with different initial configurations and the results are averaged. In the Monte Carlo simulation movements are chosen randomly such that no two events can occur at the same time. In this simulation one Monte Carlo step is equivalent to one unit of time. Total potential energy of the lattice at any instant of time when perturbed by Coulomb potential is given as

$$
E(t)=\sum_{i} \kappa_{1} \frac{1}{x_{i}} P_{i}(t),
$$

where $\kappa_{1}$ is a constant. Similarly, for the simulations that use attractive Lennard-Jones potential as the perturbation, the instantaneous potential energy of the lattice is given by

$$
E(t)=\sum_{i} \kappa_{2} \frac{1}{x_{i}^{6}} P_{i}(t),
$$

where $\kappa_{2}$ is a constant.

For comparison of relaxation function for different system sizes, we calculate dimensionless energy relaxation function $S(t)$, which can be defined as

$$
S(t)=\frac{E(t)-E(\infty)}{E(0)-E(\infty)},
$$

where $E(t)$ is the instantaneous energy at time $t$ and $E(\infty)$ is the average energy of the lattice, in equilibrium with perturbation. Density relaxation is measured in terms of a dimensionless quantity

$$
C(t)=\frac{X(t)-X(\infty)}{X(0)-X(\infty)}
$$

where $X(t)$ is defined as $X(t)=\Sigma_{i} x_{i} P_{i}(t)$.

In the equilibrium simulation the system is allowed to equilibrate with perturbation for 10000 steps to get the initial configuration. The fluctuations in total energy of the system can be defined as $F(t)=\langle E(0) E(t)\rangle$. Here a dimensionless energy fluctuation relaxation function can be defined as

$$
S(t)=\frac{F(t)-F(\infty)}{F(0)-F(\infty)} .
$$

\section{RESULTS AND DISCUSSIONS}

\section{A. Relaxation under Coulomb potential}

Figure 2 shows the energy relaxation function for different system sizes obtained from the nonequilibrium simulations of a one-dimensional channel without particle fluctuation. At short times, the potential energy of the lattice relaxes faster, as a response to the newly created center of perturbation. This results in the accumulation of random walkers near the center of the biasing field, which slows down the relaxation rate. At the same time, channels far from the center of perturbation remain active due to the reduction in the density of particles in that region. These effects together contribute to the observed slowing down of the relaxation process after the fast initial decay. As the system size increases the lowest possible energy accessible to the system obviously become lower, hence the relaxation becomes progressively slow as the system size increases. However, this effect becomes insignificant beyond a limiting size of the lattice beyond which the strength of perturbation becomes negligible. To analyze the behavior of relaxation functions in the system, we have fitted the relaxation function to a sum of an exponential and a stretched exponential of the form

$$
f_{1}(t)=b_{1} e^{-t / \tau_{1}}+b_{2} e^{-\left(t / \tau_{2}\right)^{\beta}}
$$




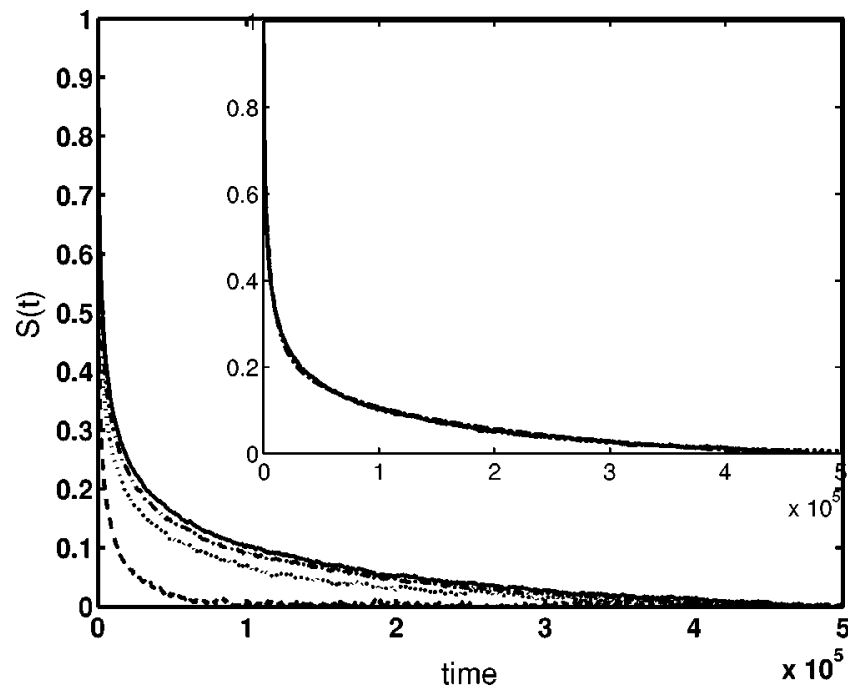

FIG. 2. Energy relaxation function $S(t)$ is plotted for four different system sizes under Coulomb potential: $\mathcal{N}_{L}=50$ (dashed line), $\mathcal{N}_{L}=100$ (dotted line), $\mathcal{N}_{L}=150$ (dashed-dotted line), and $\mathcal{N}_{L}=200$ (continuous line)in a closed system. The inset shows the fit of the energy relaxation function for $\mathcal{N}_{L}=200$ using function $f_{1}(t)$ (dotted line) and $f_{2}(t)$ (dashed-dotted line).

(with constraints $b_{1}+b_{2}=1$ and $0 \leqslant b_{1}, b_{2} \leqslant 1$ ). In order to compare with the nature of relaxation with the results obtained by Bruns et al., ${ }^{1}$ a logarithmic function of the form

$$
f_{2}(t)=1-c_{1} \log _{10}(a t)+c_{2} e^{-t / \gamma}
$$

is also used to fit the relaxation function. The inset of Fig. 2 shows the fit of the energy relaxation function with $f_{1}(t)$ and $f_{2}(t)$, when size of the system is $\mathcal{N}_{L}=200$. Fitting parameters obtained for the function $f_{1}(t)$ are $b_{1}=0.20, \tau_{1}=1.4$ $\times 10^{5}, \tau_{2}=3.4 \times 10^{5}, \beta=0.52$. The fitting parameters for the function $f_{2}(t)$ are $c_{1}=0.16, a=2.5, c_{2}=0.25$, and $\gamma=7.1$ $\times 10^{3}$. It is evident from the figure that the stretched exponential function and logarithmic functions both give good description of the energy relaxation function in a onedimensional lattice without density fluctuations, in the presence of the Coulomb potential. The time dependence of stretched exponential ${ }^{18,19}$ relaxation is given by the function of the form $S(t)=S_{0} e^{-\left(t / \tau_{s}\right)^{\beta}}$, where $0<\beta<1$. Theoretical explanation of the origin of stretched exponential relaxation in the condensed matter has been addressed by many, ${ }^{20-23}$ including Huber and co-workers in a series of papers. ${ }^{24,25}$ Their model is based on the following simple master equation approach:

$$
\frac{d P_{i}(t)}{d t}=\sum_{m \neq i} w_{m, i} P_{m}(t)-w_{i, m} P_{i}(t),
$$

where $w_{m, i}$ gives the transition probability of the particle from site $m$ to $i$. Stretched exponential relaxation can arise when there is a continuum of relaxation channels and the probability of any single channel being open is much less than unity.

Figure 3 shows the density relaxation function (which is a measure of particle aggregation) for different sizes of a system without number density fluctuations. At $\mathcal{N}_{L}=200$, a double exponential

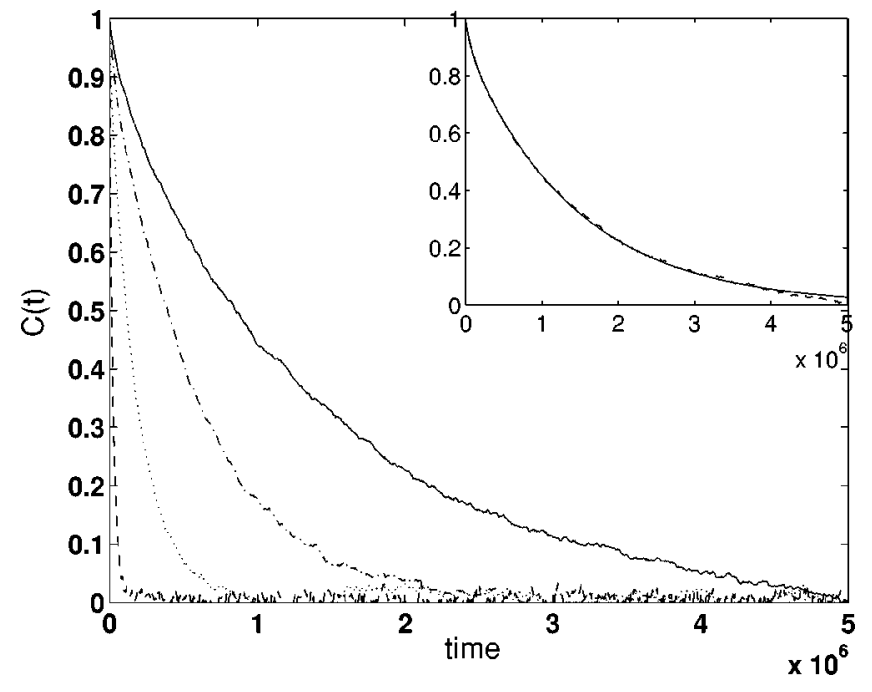

FIG. 3. The density relaxation function $C(t)$ is plotted for four system sizes under Coulomb potential: $\mathcal{N}_{L}=50$ (dashed line), $\mathcal{N}_{L}=100$ (dotted line), $\mathcal{N}_{L}=150$ (dashed-dotted line), and $\mathcal{N}_{L}=200$ (continuous line). The inset shows double exponential fit (continuous line) for $C(t)$ at $\mathcal{N}_{L}=200$.

$$
f_{3}(t)=c_{1} e^{-t / \gamma_{1}}+c_{2} e^{-t / \gamma_{2}}
$$

(with constraints $c_{1}+c_{2}=1$ and $0 \leqslant c_{1}, c_{2} \leqslant 1$ ) (shown in the inset of Fig. 3) fit of the density relaxation function reveals the presence of two distinct time scales in the density relaxation. The fitting parameters obtained for the double exponential $\left[f_{3}(t)\right]$ fit of density relaxation function are $c_{1}$ $=0.90, \gamma_{1}=1.4 \times 10^{6}, \gamma_{2}=8.3 \times 10^{4}$.

We now turn to the case when the number of walkers in the lattice can fluctuate due to the exchange with the bath. Figure 4 gives the energy relaxation function plotted for different system sizes for this case. Note that, in this case, the energy relaxation is faster than the nonfluctuating case. The exchange of particles with bath is equivalent to opening up of many wider channels of relaxation that dominate the re-

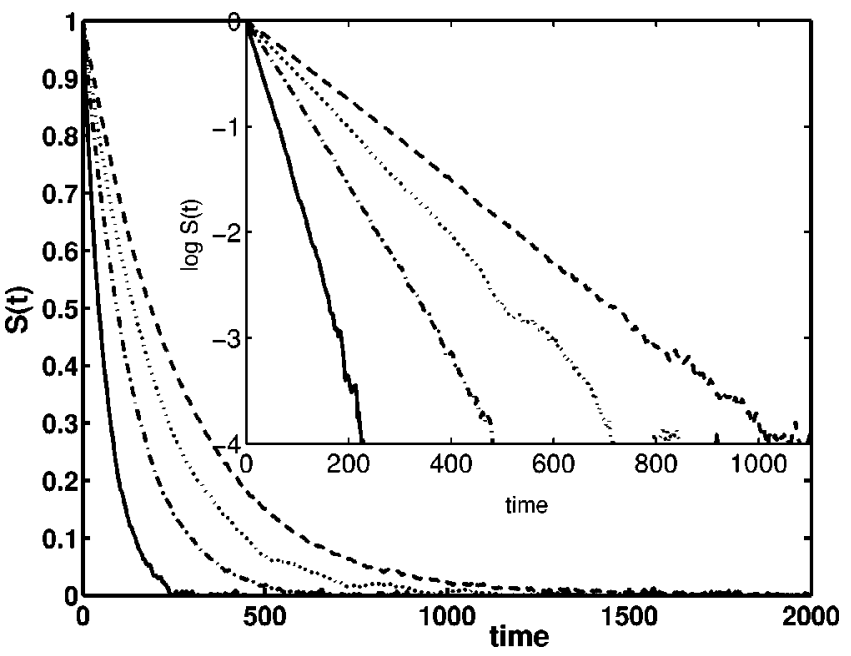

FIG. 4. Energy relaxation function is plotted for different system lengths $\left[\mathcal{N}_{L}=50\right.$ (continuous line), $\mathcal{N}_{L}=100$ (dashed-dotted line), $\mathcal{N}_{L}=150$ (dotted line), and $\mathcal{N}_{L}=200$ (dashed line)] under the perturbation of Coulomb potential. The $\log$ of $S(t)$ vs $t$ plot in the inset shows straight lines due to the exponential relaxation. 


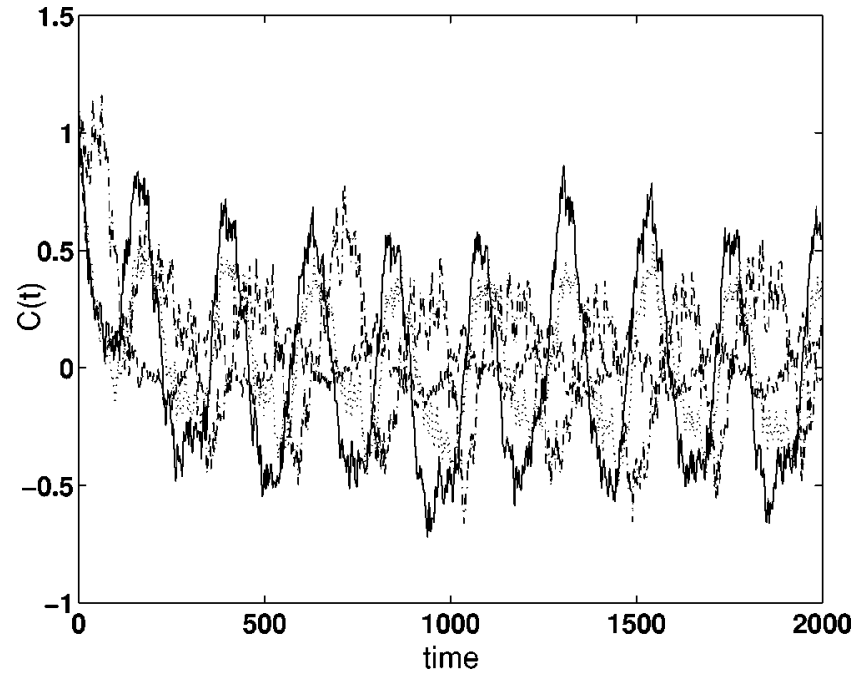

FIG. 5. Density relaxation function with density fluctuation $C(t)$ is plotted here for four system sizes $\left[\mathcal{N}_{L}=50\right.$ (continuous line), $\mathcal{N}_{L}=100$ (dasheddotted line), $\mathcal{N}_{L}=150$ (dotted line), and $\mathcal{N}_{L}=200$ (dashed line) $]$ under Coulomb potential.

laxation process. The $\log S(t)$ vs $t$ plot in the inset of Fig. 4 shows straight lines, which is an evidence of exponential relaxation. In this case walkers can bypass the obstacle caused by hard rod interaction in the path by exchange of particles with the bath. Figure 5 shows the density relaxation function of an open system for different sizes. As system size increases, the effect of Coulomb field on the distribution of carriers decreases. Hence the carrier density oscillates around a mean value due to the density fluctuations and the effect of perturbation in the density relaxation remains feeble and short lived.

The energy fluctuation relaxation function for different sizes of a system with constant number density and that is in equilibrium with perturbation is shown in the Fig. 6. This relaxation function shows very slow nonexponential decay. The inset of Fig. 6 shows the energy relaxation function obtained is well fitted by $f_{1}(t)$ and $f_{2}(t)$. The fitting parameters obtained for the $f_{1}(t)$ are $b_{1}=0.2, \tau_{1}=3.7 \times 10^{3}, \tau_{2}$ $=3.6 \times 10^{4}, \beta=0.34$ and the fitting parameters obtained for $f_{2}(t)$ are $c_{1}=0.21, a=0.06, c_{2}=0.13$, and $\gamma=6.9 \times 10^{3}$. Note that the energy relaxation function in nonequilibrium and energy fluctuation relaxation function in equilibrium show logarithmic time dependence.

\section{B. Relaxation under Lennard-Jones potential}

The pronounced nonexponential decay found in nonequilibrium energy relaxation function for Coulomb potential is also found in the case of attractive Lennard-Jones potential. Figure 7 shows the energy relaxation function at different sizes for a system without number fluctuation perturbed by the attractive Lennard-Jones potential. The fit of the energy relaxation function with $f_{1}(t)$ and $f_{2}(t)$ is shown in the inset of Fig. 7. The fitting parameters obtained for $f_{1}(t)$ are $b_{1} \simeq 0, b_{2} \simeq 1.0, \tau_{s}=1.1 \times 10^{3}, \beta=0.62$, and $f_{2}(t)$ are $c_{1}$ $=0.14, a=5.6 \times 10^{1}, c_{2}=0.58$, and $\gamma=1.3 \times 10^{3}$. It is evident from the figure that, in the case of short-ranged interaction, the appropriate function which can fit relaxation func-

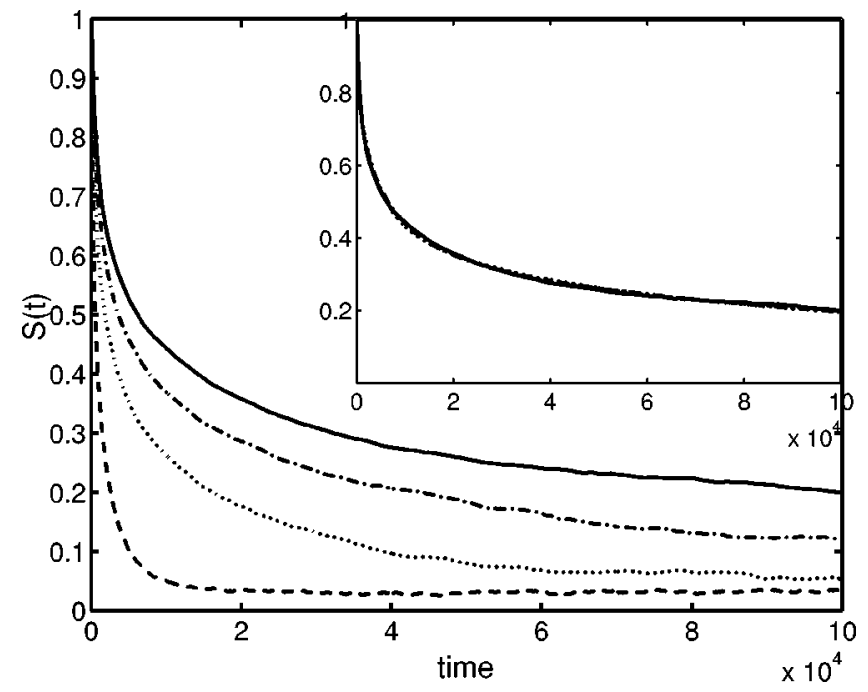

FIG. 6. Energy fluctuation relaxation function is plotted for four system sizes $\left[\mathcal{N}_{L}=50\right.$ (continuous line), $\mathcal{N}_{L}=100$ (dashed-dotted line), $\mathcal{N}_{L}=150$ (dotted line), and $\mathcal{N}_{L}=200$ (dashed line)] in equilibrium under the perturbation of Coulomb potential. The inset shows the fit of the energy relaxation function for $\mathcal{N}_{L}=200$ using function $f_{1}(t)$ (dotted line) and $f_{2}(t)$ (dasheddotted line).

tion is stretched exponential. Here due to the large strength of the potential near the perturbation center, the initial relaxation is driven and faster. Corresponding density relaxation function for different sizes of this system is shown in Fig. 8.

The energy relaxation function $[S(t)$ vs $t]$ of a linear lattice with particle number fluctuation is plotted for different system sizes in Fig. 9. The inset of Fig. 9 shows $\log$ of $S(t)$ vs $t$ plot of the energy relaxation function, which show straight lines that is a signature of exponential relaxation. It is clear from Figs. 4 and 9 that the number fluctuation in the system makes the energy relaxation nearly exponential irrespective of the nature and range of the perturbing potential.

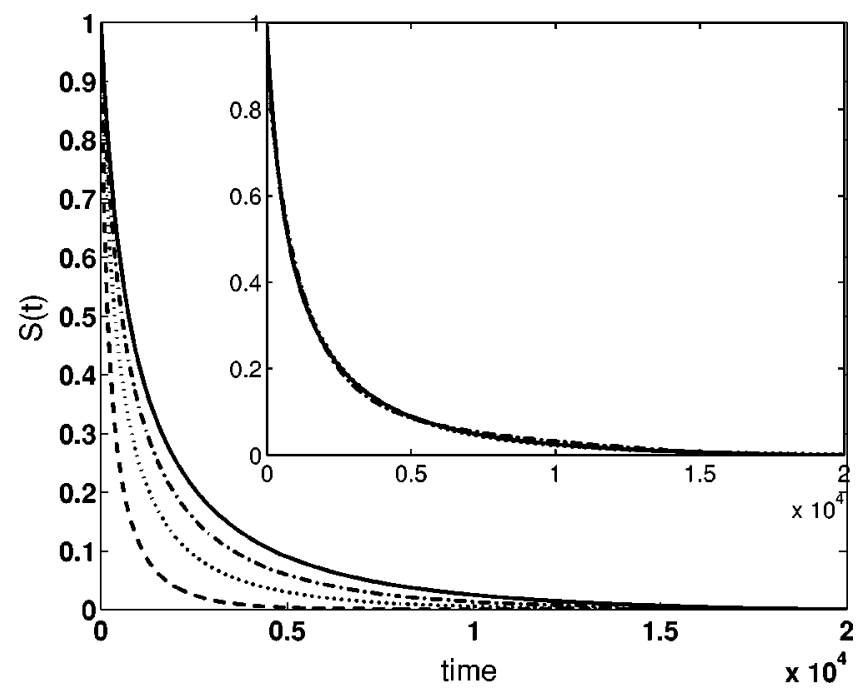

FIG. 7. Dimensionless energy relaxation function $S(t)$ of a one-dimensional channel with constant number density at different system sizes $\left[\mathcal{N}_{L}=50\right.$ (continuous line), $\mathcal{N}_{L}=100$ (dashed-dotted line), $\mathcal{N}_{L}=150$ (dotted line), and $\mathcal{N}_{L}=200$ (dashed line)] under the perturbation of Lennard-Jones potential is shown here. The inset shows the fit of the energy relaxation function for $\mathcal{N}_{L}=200$ using function $f_{1}(t)$ (dotted line) and $f_{2}(t)$ (dashed-dotted line). 


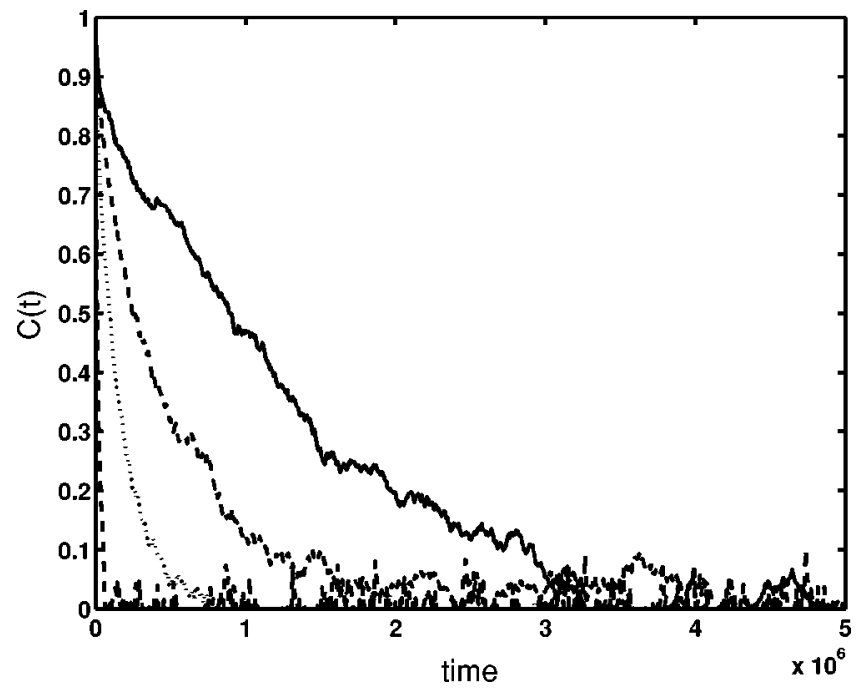

FIG. 8. Density relaxation function of the lattice under the perturbation of Lennard-Jones potential at different system sizes $\left[\mathcal{N}_{L}=50\right.$ (continuous line), $\mathcal{N}_{L}=100$ (dashed-dotted line), $\mathcal{N}_{L}=150$ (dotted line), and $\mathcal{N}_{L}=200$ (dashed line)] are shown.

The equilibrium energy fluctuation relaxation function of a system under the Lennard-Jones perturbation, for different system sizes, is plotted in Fig. 10. The energy fluctuation in this system is mostly from the region where potential is weak. The fit of the energy fluctuation relaxation with functions $f_{1}(t)$ and $f_{2}(t)$ is shown in the inset of Fig. 10. The fitting parameters for the function $f_{1}(t)$ are $b_{1}=0.42, \tau_{1}$ $=8.8 \times 10^{3}, \tau_{2}=1.6 \times 10^{5}, \beta=0.73$, and for $f_{2}(t)$ are $c_{1}$ $=0.14, a=5.6 \times 10^{1}, c_{2}=0.58$, and $\gamma=1.3 \times 10^{3}$.

Finally, note that the stretched exponential fit of relaxation function is more appropriate in this case due to the short range of the potential. We have found no evidence of logarithmic time dependence for the energy relaxation in this case.

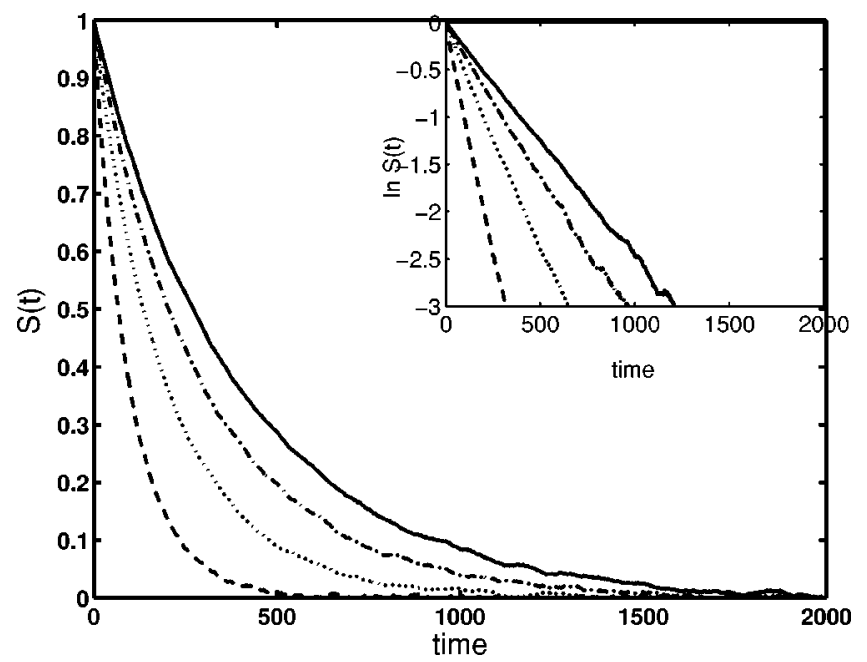

FIG. 9. Energy relaxation function $[S(t)]$ is plotted at different system sizes $\left[\mathcal{N}_{L}=50\right.$ (continuous line), $\mathcal{N}_{L}=100$ (dashed-dotted line), $\mathcal{N}_{L}=150$ (dotted line), and $\mathcal{N}_{L}=200$ (dashed line)] with particle number fluctuations under Lennard-Jones potential. The $\log$ of $S(t)$ vs $t$ plot in the inset shows straight lines due to the exponential relaxation.

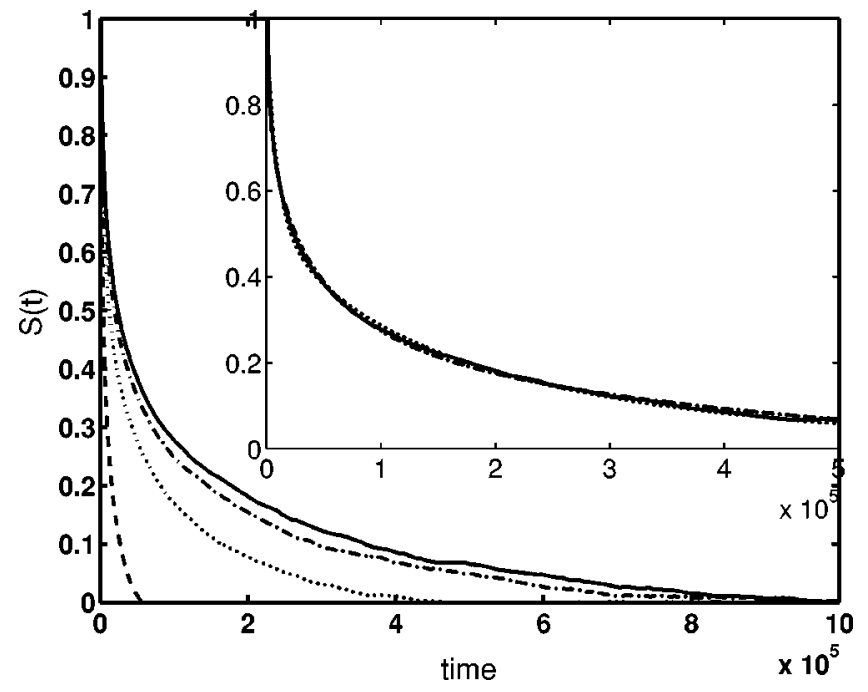

FIG. 10. Energy fluctuation relaxation function is plotted for system in equilibrium under the perturbation of Lennard-Jones potential for different system sizes $\left[\mathcal{N}_{L}=50\right.$ (continuous line), $\mathcal{N}_{L}=100$ (dashed-dotted line), $\mathcal{N}_{L}$ $=150$ (dotted line), and $\mathcal{N}_{L}=200$ (dashed line)]. The inset shows the fit of the energy relaxation function for $\mathcal{N}_{L}=200 \mathrm{using}$ function $f_{1}(t)$ (dotted line) and $f_{2}(t)$ (dashed-dotted line).

\section{CONCLUSION}

Let us first summarize the main results of this work. We have demonstrated that the cooperative dynamics of random walkers in a simple one-dimensional channel can give rise to highly nonexponential relaxation, when number density fluctuations are not allowed. In these simulations, two perturbing potentials (the Coulomb and the Lennard-Jones) have been used to study the effects of perturbing potential on the relaxation process. The energy relaxation under these potentials can be approximately described by a stretched exponential (in general) in a closed system. The variation in the time scale of relaxation under these two well-known potentials can be understood in terms of the difference in the range of these potentials. The Coulomb potential being long ranged (in comparison with the Lennard-Jones interaction), shows much stronger nonexponentiality in the energy relaxation. Under the Coulomb potential, the exponent $\beta$ of nonequilibrium energy relaxation function is 0.52 while under LennardJones potential it is 0.73 . The simulations seem to agree with the results of Bruns et al. ${ }^{1}$ in showing a logarithmic time dependence of the energy relaxation function under the Coulomb potential. However, under the short-ranged LennardJones potential, the energy relaxation function does not show logarithmic time dependence.

In the smaller sized systems the relaxation is found to be faster and as the system size increases, relaxation slows down, as expected. Also as expected, the density fluctuations in this one-dimensional channel make the relaxation function faster and exponential. When number fluctuation is allowed, random walkers overcome the resistance of the hard rod interactions, by moving in and out of the linear lattice, such that the random walkers experience no major hindrance to their flow. In this case the system behaves like a system of weakly interacting particles, without the need for strong cooperativity for relaxation. It is worth noting that interactions 
found in nature are numerous, but the models of asymptotic time dependence of relaxation commonly found in nature are limited in number. However, the parameters of the relaxation function depend on the form of the biasing potential and the nature of interaction between the carriers.

\section{ACKNOWLEDGMENTS}

This work was supported in part by grants from the Department of Atomic Energy (DAE) and the Council of Scientific and Industrial Research (CSIR), India.

${ }^{1}$ E. B. Bruns, M. L. Madaras, R. S. Coleman, C. J. Murphy, and M. A. Berg, Phys. Rev. Lett. 88, 158101 (2002).

${ }^{2}$ S. K. Pal, L. Zhao, T. Xia, and A. H. Zewail, Proc. Natl. Acad. Sci. U.S.A. 100, 13746 (2003).

${ }^{3}$ S. Chandrasekhar, Rev. Mod. Phys. 15, 1 (1943).

${ }^{4}$ Non-equilibrium Statistical Mechanics in One Dimension, edited by V. Privman (Cambridge University Press, Cambridge, 1997).

${ }^{5}$ S. Alexander, J. Bernasconi, W. R. Schneider, and R. Orbach, Rev. Mod. Phys. 53, 175 (1981).

${ }^{6}$ E. Barkai, V. Fleurov, and J. Klafter, Phys. Rev. E 61, 1164 (2000).
${ }^{7}$ D. L. Huber, Phys. Rev. B 15, 533 (1977).

${ }^{8}$ P. M. Richards, Phys. Rev. B 16, 1393 (1977).

${ }^{9}$ P. M. Richards and R. L. Renken, Phys. Rev. B 21, 3740 (1980).

${ }^{10}$ K. W. Yu and P. M. Hui, Phys. Rev. A 33, 2745 (1986).

${ }^{11}$ R. Pitis, Phys. Rev. B 48, 4196 (1993).

${ }^{12}$ G. Zumofen and J. Klafter, Phys. Rev. E 51, 2805 (1995).

${ }^{13}$ A. Bar-Haim and J. Klafter, J. Chem. Phys. 109, 5187 (1998).

${ }^{14}$ C. Rodenbeck, J. Karger, and K. Hahn, Phys. Rev. E 55, 5697 (1997).

${ }^{15}$ P. H. Nelson and S. M. Aurbach, J. Chem. Phys. 110, 9235 (1999).

${ }^{16}$ M. S. Okino, R. Q. Snurr, H. H. Kung, J. E. Ochs, and M. L. Mavrovouniotis, J. Chem. Phys. 111, 2210 (1999).

${ }^{17}$ D. Frenkel and B. Smith, Understanding Molecular Simulation: From Algorithms to Applications (Academic, San Diego, 1996).

${ }^{18}$ R. Klohlrausch, Ann. Phys. (Leipzig) 12, 393 (1847).

${ }^{19}$ G. Williams and D. C. Watts, Trans. Faraday Soc. 66, 80 (1970).

${ }^{20} \mathrm{~W}$. Gotze, in Liquid Freezing and Glass Transition, edited by D. Levesque, J. P. Hansen, and J. Zinn-Justin (North Holland, Amsterdam, 1990).

${ }^{21}$ W. Gotze and L. Sjogren, Rep. Prog. Phys. 55, 241 (1992).

${ }^{22}$ M. O. Vlad and M. C. Mackey, J. Math. Phys. 36, 1834 (1995).

${ }^{23}$ M. W. Cohen and G. S. Grest, Phys. Rev. B 24, 4091 (1981).

${ }^{24}$ D. L. Huber, D. S. Hamilton, and B. Barnett, Phys. Rev. B 16, 4642 (1977).

${ }^{25}$ D. L. Huber, Phys. Rev. B 31, 6070 (1985); Phys. Rev. E 53, 6544 (1996). 